\title{
Montelukast treatment (cysteinyl leukotriene receptor antagonist) in a model of food allergy: modifications in lymphatic cell population from rectal mucosa
}

\author{
M. Vinuesa, N. Bassan, A. I. Cases and G. Krumrik \\ Histology and Embriology Department. School of Medical Sciences. Universidad Nacional de Rosario. Rosario, \\ Argentina
}

\begin{abstract}
Objective: the aim is to determine immunopathological modifications in rectal mucosa from rabbits after local challenge in ovalbumin (OVA) sensitized animals previously treated with montelukast.

Material and methods: experimental design: thirty two rabbits divided into four groups: G1: normal; G2: subcutaneously OVA sensitized; G3: sensitized, locally OVA challenged and sampled 4 hours after challenge; and G4: sensitized, locally OVA challenged and treated 4 hours before challenge with montelukast $(0.15 \mathrm{mg} / \mathrm{kg})$. Specific anti-OVA IgE levels were evaluated by passive cutaneous anaphylaxis test (PCA). In each group 200 high microscopical power fields (HPF) were counted. Results were expressed as arithmetic mean and SE. Anti -CD4, CD5, $\mu$ chain monoclonal antibodies were used. Avidin biotin horseradish peroxidase system was used.

Results: $C D$ 4: G1: $8.3 \pm 0.06$; G2: $13.4 \pm 0.08, \mathrm{G} 3: 8.25$ \pm 0.06 , G4: $11.8 \pm 0.02$. CD 5: G1: $7.3 \pm 0.05$; G2: $9.4 \pm$ 0.05, G3: $11.3 \pm 0.06$, G4: $8.1 \pm 0.06 . \mu$ chain: G1: $10.4 \pm$ $0.06 ; \mathrm{G} 2: 3.8 \pm 0.02, \mathrm{G} 3: 6.0 \pm 0.10, \mathrm{G} 4: 2.2 \pm 0.10$. In all cases, experimental groups (G3 vs. G4) presented statistical significant differences ( $p<0.05)$. CD4+, CD5+ cells and $\mu$ chain + decrease in experimental group (G4), probably due to lymphocyte migration inhibition to challenged mucosa. $\mu$ chain + cell decrease could be based on B cell activation and expression of different surface immunoglobulins. Cells expressing $\mu$ chain decreased in G2 and G3 likely due to activation of B cells and subsequent expression of other immunoglobulin chains in cell surface.

Conclusions: we conclude that obtained data are important to elucidate immunopathology of local anaphylactic reaction in rectal mucosa from systemic sensitized animals after treatment with montelukast.
\end{abstract}

Key words: Immunpathology. Rectal mucosa. Rabbit. Food allergy. Montelukast.

Received: 24-04-09.

Accepted: 09-02-10.

Correspondence: Miguel Vinuesa. Superi 247. Rosario (2000) Santa Fe, Argentina.e-mail: vinuesamiguel@gmail.com
Vinuesa M, Bassan N, Cases AI, Krumrik G. Montelukast treatment (cysteinyl leukotriene receptor antagonist) in a model of food allergy: modifications in lymphatic cell population from rectal mucosa. Rev Esp Enferm Dig 2010; 102: 421-425.

\section{INTRODUCTION}

Montelukast is a cysteinyl-leukotriene receptor antagonist (CysLT) which actively and selectively blocks the leukotriene D4 (LTD4) receptor, which has shown an inhibition of allergy response in different animal and human models (1-6).

It has been established that leukotrienes participate in the allergic inflammatory phenomena induced by food allergy, which is described as an anomalous immune response developed in previously sensitized individuals, when incorporating through the digestive tract substances that contain the allergen to which they are sensitized (79).

Food allergy is a common widespread pathology with an increasing prevalence (10). Very few drugs have been shown to be effective in treating or preventing clinical symptoms of food allergy. Allergens may be normal compounds of nutrients, additives and transgenic variety of natural products. The way of cooking may not only increase or decrease the antigenic properties of food but also modify molecular structures, inducing cross-reactions (11).

Among the most studied foods capable of producing allergy are: cow milk, chicken egg, wheat, tomato, chocolate, peanut, kiwifruit, fish, oysters and shellfish (12-14).

In the digestive tract, food antigens may be incorporated through an appropriate penetration of molecules and 
may induce a tolerance response (10). Moreover, they may enter in an inappropriate form (altered mucosal barrier), which induces sensitization and high levels of specific IgE, which binds mast-cells and basophils (15-17).

The ingestion of food antigen in sensitized individual triggers the development of an acute local anaphylactic inflammatory reaction (18). This reaction induces qualitative modifications of the mucosa and quantitative changes in lymphatic cells populations (19).

The potential for animal models to mimic the human disease process makes them an attractive tool for determining disease mechanisms, predicting disease triggers, and testing treatment regimens. With this in mind, animal models of food allergy have been receiving increasing attention as research tools to answer some of the difficult questions regarding food-allergy disease (20).

The rabbit constitutes a very useful biological model for studying food allergy. It is a lagomorph that has an important genetic preservation with human genome (21). It generates high levels of IgE after subcutaneous sensitization (22-24). Moreover, a high amount of potentially antigenic food macromolecules reach distal sectors of the large intestine because of the physiologic process of cecotrophy (25).

The rectum belongs to the common immune mucosal system (CIMS), which determines that the sensitization processes and post challenge with food antigens produce histopathological and immunological modifications, which may be evaluated in the rectal mucosa $(26,27)$.

The aim of the present work is to establish quantitative modifications in distribution of $\mathrm{CD} 4, \mathrm{CD} 5, \mu$ chain positive cells, mast-cells and eosinophils, which take part in the immune response at rectal mucosa, in a model of mucosal immunity and food allergy in animals treated with montelukast. Our hypothesis is that the blocking of cysteinyl-leukotrienes receptor produces an inhibition over pro inflammatory mediators that intervene in phenomena of IgE-mediated allergic inflammation in rabbit rectum.

Diagnosis and treatment of food allergy offers many complications. Furthermore, treatment is mainly design to stop symptoms instead of prevention of potential reactions. The knowledge of potential effects of $\mathrm{AL}$ at allergic inflammation phenomena in rectal mucosa let us obtain a useful tool in order to treat food allergy.

\section{MATERIALS AND METHODS}

Thirty two adult New Zealand rabbits were divided in 4 groups:

-Group 1: $(\mathrm{n}=8)$ normal control.

-Group 2: $(\mathrm{n}=8)$ ovalbumin (OVA) sensitized subcutaneously (SC).

-Group 3: $(\mathrm{n}=8)$ OVA sensitized SC and OVA rectal challenged.

-Group 4: $(\mathrm{n}=8)$ OVA sensitized SC and OVA rectal challenged previously treated (4 hours before challenge) with montelakust $(0.15 \mathrm{mg} /$ kilogram $)$ in a single dose.
As we described in previous works, rabbits from group 2 and 3 were twice subcutaneously sensitized with $70 \mu \mathrm{g}$ OVA in $30 \mathrm{mg}$ ALUM/ml (aluminium hydroxide). An interval of 15 days among sensitizations is developed. After 15 days post sensitization, rabbits were 24 hours fasten and G3 and G4 animals were rectal challenged with $50 \mathrm{mg}$ OVA in $5 \mathrm{ml}$ of PBS.

Animals were sacrificed with sulfuric ether 4 hours after challenged, according to considerations of Ethical Committee of Rosario School of Medicine. Samples from rectum were obtained from all groups.

Material for immunohistochemistry were snap frozen in liquid nitrogen with OCT protector and stored at $-20^{\circ} \mathrm{C}$ and cut at 8 micrometers.

Samples for histology were paraffin embedded and cut at 6 micrometers

The following monoclonal antibodies were used:

- Mouse anti-rabbit CD5 (KEN-5, BALB/c IgG 1, BIOSOURCE, Camarillo, California, U.S.A.), for mature total $\mathrm{T}$ cell identification and B cell subset (28).

- Mouse anti-rabbit CD4 (KEN-4, BALB/c IgG 2 a, BIOSOURCE, Camarillo, California, U.S.A) for CD4+ T cells identification (28).

- Mouse anti-rabbit immunoglobulin M (cadena $\mu$, NRBM BALB/c IgG1, BIOSOURCE, Camarillo, California, U.S.A.) B cell marker (28).

-Botin-streptavidin system (Serotec Ltd. Oxford, UK) was used for detection of marked cells and AEC as chromogen.

-Frozen samples were fixed in cold acetone for 10 minutes and then incubates 60 minutes at $37^{\circ} \mathrm{C}$ with monoclonal antibodies.

- Chromotope II technique was employed to detection of eosinophils and Alcian blue $\mathrm{pH}<1$ was used for mastcells in rectum.

- Positive cells were analyzed in 200 high power fields in each group and expressed as cell mean per microscopical field. Results were statistical analyzed.

- Specific anti-OVA IgE titres were evaluated by passive cutaneous anaphylaxis PCA $(29,30)$.

\section{RESULTS}

Specific anti-OVA-IgE levels were evaluated by positive passive cutaneous anaphylaxis test (PCA) positive at 160 fold dilutions in sensitized groups (G2, G3, G4).

Histology of samples showed patchy mucosal edema, lymphangiectasia and eosinophil infiltration in the experimental OVA sensitized and challenged groups (G3 and G4). Eosinophils were spread on the mucosa underlying the epithelium.

There were no visualized histopathological modifications in the control group (G1) and the sensitized and unchallenged group (G2).

CD4 (Fig. 1) and CD5 positive cells (Fig. 2) predominate in the lamina propria of the rectum of both control 


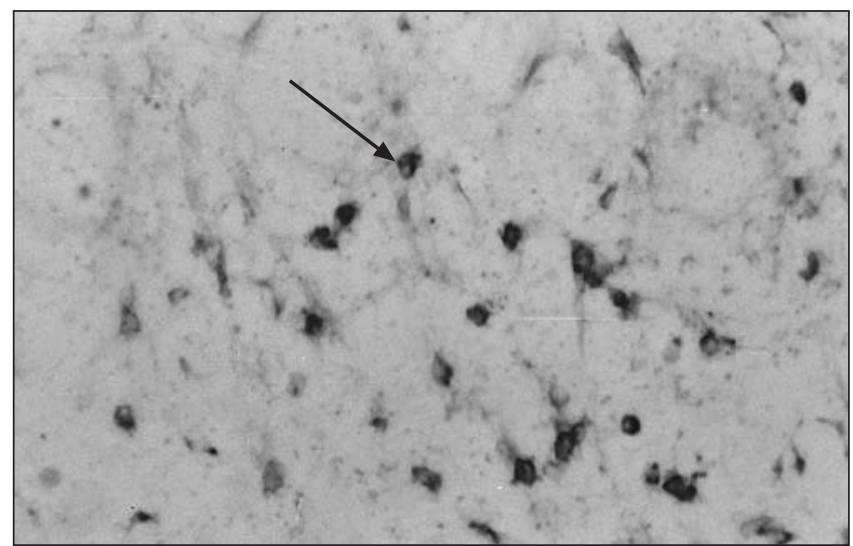

Fig. 1. CD4 positive cells in the rectal mucosa adjacent to the bottom of crypts. Sensitized and challenged rabbits. Observe the nucleus lack of staining. x 400. Group 3.

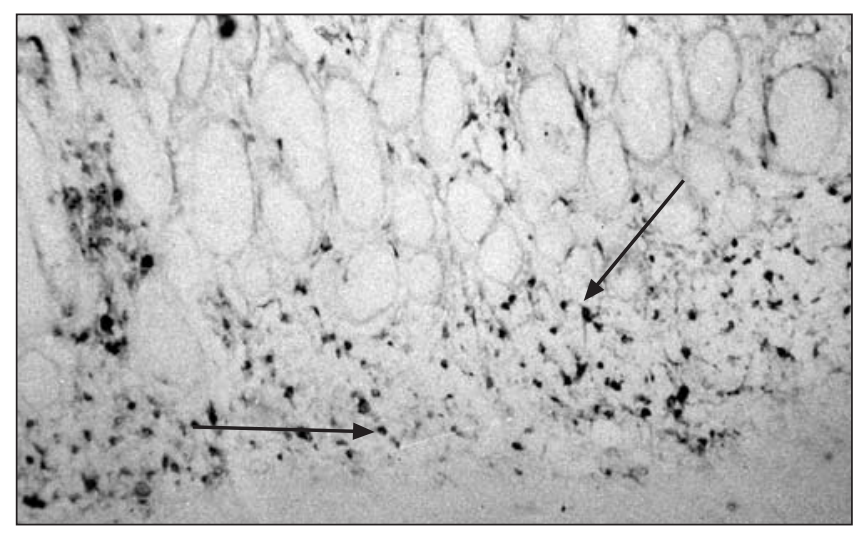

Fig. 2. CD5 positive cells in the rectal mucosa from sensitized and treated rabbits with montelukast. x 100. Group 4.

and experimental groups. It can be observed significant differences between group treated with montelukast (G4) and no-treated animals control (Table I).

CD4, CD5 and $\mu$ chain + cells presented a decrease in the group treated with montelukast, compared with animals challenged without treatment (Fig. 3).

Mast cells were increased in sensitized group showing a subsequent decrease after challenge.

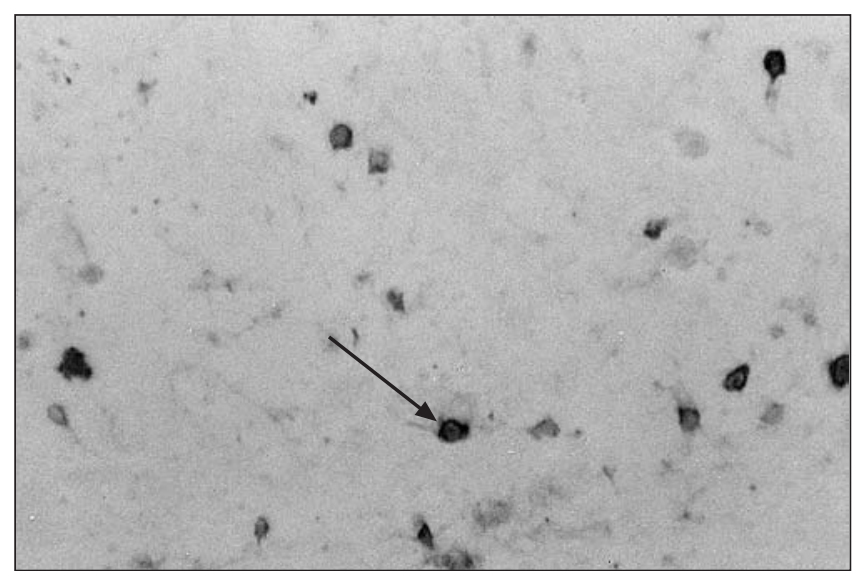

Fig. 3. Deep area of the rectal mucosa in which $\mu$ chain positive cells are observed. Sensitized and challenged rabbits. x 400. Group 3.

\section{DISCUSSION}

Ovalbumin is a soluble antigen which, when subcutaneously administered, induces sensitization that elicits specific IgE antibodies. Challenge with OVA in sensitized individuals produces an anaphylactic allergic response in the digestive tract.

In our study, we demonstrated allergic inflammation in rectal mucosa from rabbits that were subcutaneously sensitized and locally challenged with OVA (G3 and G4).

We observed eosinophil infiltration, vascular congestion and lymphangiectasia. Such signs become apparent 4 hours after OVA challenge.

Previous treatment with montelukast at fixed dose produced modifications in the studied immune cell populations with a decrease in the number of CD4 and CD5 cells in the group treated with montelukast against the group challenged without treatment. Montelukast showed to inhibit CD4 and CD5 infiltration after challenge.

The statistically significant decrease found in both cells populations could be related to an inhibition in the lymphocyte migration post challenge. In different animal and human models of acute and chronic intestinal inflam-

Table I. Cell populations in rectal mucosa from normal rabbits, ova sensitized rabbits and sensitized and locally challenged rabbits (positive cells per high power field arithmetic mean and SE)

\begin{tabular}{|c|c|c|c|c|}
\hline Markers & $\begin{array}{l}\text { G1: control } \\
n=200\end{array}$ & $\begin{array}{l}\text { G2: sensitized } \\
\quad n=200\end{array}$ & $\begin{array}{l}\text { G3: sensitized and challenged } \\
\qquad n=200\end{array}$ & $\begin{array}{l}\text { G4: sensitized and challenged, } \\
\text { treated with montelukast } \\
\qquad n=200\end{array}$ \\
\hline CD 4 & $8.3 \pm 0.06$ & $13.4 \pm 0.08$ & $16.4 \pm 0.06$ & $11.8 \pm 0.02 *$ \\
\hline CD 5 & $7.3 \pm 0.05$ & $9.4 \pm 0.05$ & $11.3 \pm 0.06$ & $8.1 \pm 0.06^{*}$ \\
\hline$\mu$ chain & $10.4 \pm 0.06$ & $3.8 \pm 0.02$ & $6.0 \pm 0.10$ & $2.2 \pm 0.10^{*}$ \\
\hline Mast cells & $8.7 \pm 3.0$ & $17.6 \pm 5.0$ & $6.0 \pm 1.8$ & $7.2 \pm 2.7 * \star$ \\
\hline Eosinophils & $0.08 \pm 027$ & $0.25 \pm 0.43$ & $1.25 \pm 0.44 \#$ & $1.33 \pm 0.47 \#$ \\
\hline
\end{tabular}

*Different from sensitized and challenged animals without treatment group (G3) $p<0.05$. **Different from $G 2(p<0.001)$; \#Different from $G 1$ and $G 2$ ( $p<0.05)$. 
mation, CD4 monoclonal antibody that is expressed on lymphocyte subpopulation of auxiliary LT and anti CD5 (recognizes total LT in rabbits) were increased as a result of an inflammatory infiltration (31).

As seen in table I, $\mu$ chain positive cells showed a significant decrease after sensitization, and the decrease is still present after challenged. This fact could be based on the expression of different surface chains, other than $\operatorname{IgM}$ due to the $\mathrm{B}$ cells activation.

Other possibility would be that of migration of B cells to other tissues.

These obtained data in rectal mucosa from the group treated with anitluekotrienes would be related to the inhibition of allergic inflammation in its effector side of the immune response.

Different authors describe inhibition of the eosinophils infiltration after the use of montelukast in different animal and human models, mainly in the respiratory system $(3,4)$. The absence of statistically significant differences in the quantity of eosinophils observed among the groups studied is probably due to different characteristics of rectal mucosa model as compared to bronchial mucosa (32,33).

Mast cells showed a significant increase in sensitized rabbits $(\mathrm{G} 2)$. The decrease in number of observed mast cells after challenge is due, as we previously determined, by a phenomena of degranulation induced by the contact with specific antigen. That mast cell loses of granules determines a decrease possibility to be observed $(34,35)$.

We conclude that treatment with montelukast in our model induced different modifications in studied cell populations in experimental groups, mainly based on inhibition of eosinophil infiltration and mast cell degranulation.

These described modifications let us establish a useful role of $\mathrm{AL}$ as preventive drug to treat already diagnosed food allergy under determined conditions. Even though this data is referred to an animal model, further studies are required for human food allergy.

However, the participation of different intercellular mediators such as interleukins, cytokines and chemokines should be analyzed in future experimental works in order to advance in the comprehension of the allergic inflammatory mechanisms and its inhibition.

\section{REFERENCES}

1. Riccioni G, Bucciarelli T, Mancini B, Di Ilio C, D’Orazio N. Antileukotriene drugs: clinical application, effectiveness and safety. Curr Med Chem 2007; 14(18): 1966-77.

2. Capra V, Ambrosio M, Riccioni G, Rovati GE. Cysteinyl-leukotriene receptor antagonists: present situation and future opportunities. Curr Med Chem 2006; 13(26): 3213-26.

3. Muz MH, Deveci F, Bulut Y, Ilhan N, Yekeler H, Turgut T. The effects of low dose leukotriene receptor antagonist therapy on airway remodeling and cysteinyl leukotriene expression in a mouse asthma model. Exp and Mol Med 2006; 38(2): 109-18.
4. Salvi SS, Krishna MT, Sampson AP, Holgate ST. The anti-inflammatory effects of leukotriene-modifying drugs and their use in asthma. Chest 2001; 119: 1533-46.

5. Drazen JM, Israel E, O'Byrne PM. Treatment of asthma with drugs modifying the leukotriene pathway. N Engl J Med 1999; 340: 197206.

6. Ducharme FM, Di Salvio F. Anti-leukotriene agents compared to inhaled corticosteroids in the management of recurrent and/or chronic asthma in adults and children. Cochrane Database Syst Rev 2004; (1): CD002314.

7. Brandtzaeg PE. Current understanding of gastrointestinal immunoregulation and its relation to food allergy. Ann N Y Acad Sci 2002; 964: 13-45.

8. Taylor SL, Hefle SL. Food science perspective on food allergy. Allergy 1998; 53: 5-7.

9. Kanaoka Y, Boyce JA. Cysteinyl leukotrienes and their receptors: cellular distribution and function in immune and inflammatory responses. J Immunol 2004; 173: 1503-10.

10. Lack G. Food allergy. NEJM 2008; 12(359): 1252-60.

11. Sabbah A, Drouet M, Luret MG, Giffard G, Minkhar M. Cross reactivity between cow's milk and goat's milk. Allerg Immunol 1997; 29: 203-14.

12. Ahmed T, Fuchs G. Gastrointestinal allergy to food: a review. J Diarrheal Dis Res 1997; 15: 211-23.

13. Ramírez Cruz NE, Castrejón Vázquez MI, Espinoza Goldman MB, Martínez-Cairo Cueto S. Prevalence of latex-fruit syndrome in health workers with latex allergy. Rev Alerg Mex 2002; 49: 46-51.

14. Helm RM, Burks AW. Food allergens. Clin Allergy Immunol 2008; 21: 219-35.

15. van Ree R, Akkerdaas J, van Leeuwen A, Fernández Rivas M, Asero $\mathrm{R}, \mathrm{Knul}-\mathrm{Bretlova} \mathrm{A}$, et al. New perspectives for the diagnosis of food allergy. Allergy Clin Immunol Int 1998; 12: 7-12.

16. Sampson HA. Food allergy. JAMA 1999; 278: 1888-94.

17. Placenti G, Bertolini A, Spezia E, Piscione T, Boner AL. Ability of new infants formula prepared from partially hydrolysed whey to induce anaphylactic sensitization: evaluation in guinea pig model. Allergy 1994; 49: 361-4.

18. Miller K, Laugee J, Meredith C. The monitoring of effects of food components on immunoreactivity in experimental animals. Allergy 1998; 53: 35-7.

19. Vinuesa M, Tanaka Y, Hakugawa J, Jae Bae S, Katayama I. In-situ expression of interleukin-4, 5 and 6 in Peyer's patch from Ovalbumin (OVA)-sensitized BALB/c mice after oral challenge. Int Allergol 1997; 46: 243-7.

20. McClain S, Bannon GA. Animal models of food allergy: opportunities and barriers. Curr Allergy Asthma Rep 2006; 6(2): 141-4.

21. Mage RG. Immunology of lagomorphs. En: Pastoret P, Griebel P, Bazin H, Govaerts A, editores. Handbook of vertebrate immunology. San Diego: Academic Press; 1998. p. 223-60.

22. Fekete $\mathrm{S}$, Bonori J. The effect of the fiber and protein level of the ration upon the cecotrophy of rabbit. J Appl Rabbit Res 1985; 8: 68-71.

23. Hollyster AG. Effects of water administrated probiotics and acidifiers on growth feed conversion and enteritis mortality of weaning rabbit. J Appl Rabbit Res 1989; 12: 143-7.

24. Fekete S. Recent finding and future perspectives of rabbit's digestive physiology. Cuni Sciences 1987; 4: 1-9.

25. Bassan N, Vinuesa M, Roma S, Pérez F. Biological model for detection of food antigens. Arch Latinoam Nutr 2002; 52: 249-56.

26. Cellier C, Cervoni JP, Leborgne M, Marteau P, Landi B, Cerf-Bensussan N, et al. Gluten-free diet induces regression of T-cell activation in the rectal mucosa of patients with celiac disease. Am J Gastroenterol 1998; 93: 1527-30.

27. Ensari A, Marsh M, Loft D, Morgan S. Moriarty, K. Morphometric analysis of intestinal mucosa. V-quantitative histologic and immunocytochemical studies of rectal mucosae in gluten-sensitivity. Gut 1993; 34: 1225-9.

28. Kotani M. Generation and characterization of monoclonal antibodies against rabbit CD4, CD5 and CD11a antigens. J Imm Meths 1993; 157: 241-52.

29. Bassan N, Vinuesa M, Pérez F, Roma S, Bernardi S, Lagrutta M. Mastocitos azul alciano positivos en la mucosa del ciego de conejos normales y esplenectomizados, sensibilizados y desafiados con ovoalbúmina. Analecta Veterinaria 1998; 18: 15-20. 
30. Kanaoka Y, Maekawa A, Penrose JF, Austen KF, Lam BK. Attenuated zymosan-induced peritoneal vascular permeability and IgE-dependent passive cutaneous anaphylaxis in mice lacking leukotriene C4 synthase. J Biol Chem 2001; 276: 22608-13

31. Paruchuri S, Mezhybovska M, Juhas M, Sjolander A. Endogenous production of leukotriene D4 mediates autocrine survival and proliferation via CysLT1 receptor signaling in intestinal epithelial cells. Oncogene 2006; 25: 6660-5.

32. Rothenberg ME, Hogan SP. The eosinophil. Annu Rev Immunol 2006; 24: 147-74.
33. Bandeira-Melo C, Weller PF. Eosinophils and cysteinyl leukotrienes. Prostaglandins Leukot Essent Fatty Acids 2003; 69: 135-43.

34. Mellor EA, Maekawa A, Austen KF, Boyce JA. Cysteinyl leukotriene receptor 1 is also a pyrimidinergic receptor and is expressed by human mast cells. Proc Natl Acad Sci USA 2001; 98: 7964-9.

35. Jiang Y, Borrelli LA, Kanaoka Y, Bacskai BJ, Boyce JA. CysLT2 receptors interact with CysLT1 receptors and down-modulate cysteinyl leukotriene-dependent mitogenic responses of human mast cells. Blood 2007; 110: 3263-70. 